\title{
LICENSE PLATE LOCALIZATION USING GABOR FILTERS AND NEURAL NETWORKS
}

\author{
Sami Ktata, Faouzi Benzarti and Hamid Amiri \\ Department of Electrical Engineering, \\ Signal, Image and Information Technologies Research Laboratory (LR-SITI), \\ National Engineering School of Tunis, Tunis El Manar University, Tunis-Belvedere, Tunisia
}

Received 2013-07-01, Revised 2013-08-24; Accepted 2013-08-28

\begin{abstract}
Vehicle License Plate Detection (LPD) is an important step for the vehicle plate recognition which can be used in the intelligent transport systems. Many methods have been proposed for the detection of license plates based on: Mathematical morphology, Discrete Wavelet Transform, Hough Transform and others. In general, an LPR system includes four main parts: Vehicle image acquisition, license plate detection, character segmentation and character recognition. In this study, we present a robust method for extracting and detecting license plates, from simple images of Tunisian vehicles, based on Gabor filters and neural networks. The proposed method is designed to perform recognition of any kind of license plates under any environmental conditions.
\end{abstract}

Keywords: Gabor Filters, Neural Network, Plate Detection, Contrast Limited Adaptive Histogram Equalization, Wavelets, Fast Fourier Transform

\section{INTRODUCTION}

To track and analyze vehicles motion, several intelligent transport systems have been developed to simplify the problem of identification of vehicles through various techniques which usually support on automated algorithms. The license plate can be considered as the most important for the vehicles identification. Indeed, the recognition of license plate covers many applications such as supervision systems and parking management systems. The need for automatic license plate recognition is due to several disadvantages of manual systems. The challenge is to built a robust system which meet several complex image processing conditions such as brightness, complex background, the deficiency of the plate (damaged or dirty)... In addition, it attempts to deal with fraud or theft, the use of robust systems of recognition of license plates is a necessity.
Generally, an LPR system includes four main parts which are represented as Fig. 1.

The LP detection step enables the classification of the non LP and the LP regions. This step is considered as the most important one, insofar as a good localization leads to a high accuracy and a real time segmentation and recognition.

The characters segmentation step separates characters from others in a LP so that only the precise contours of each character image block is kept for recognition. Finally the character recognition step converts images based on predefined recognition models.

However, LPR technique is still evolving through various requirements; the most common is the variation of styles LP, for example, the appearance variation caused by changes in the conditions of image capture, style change plate. The objective of this study is to implement a robust method using Gabor filters for feature extraction and neural networks for plate recognize.

Corresponding Author: Sami Ktata, Department of Electrical Engineering, Signal, Image and Information Technologies Research Laboratory (LR-SITI), National Engineering School of Tunis, Tunis El Manar University,

Tunis-Belvedere, Tunisia 


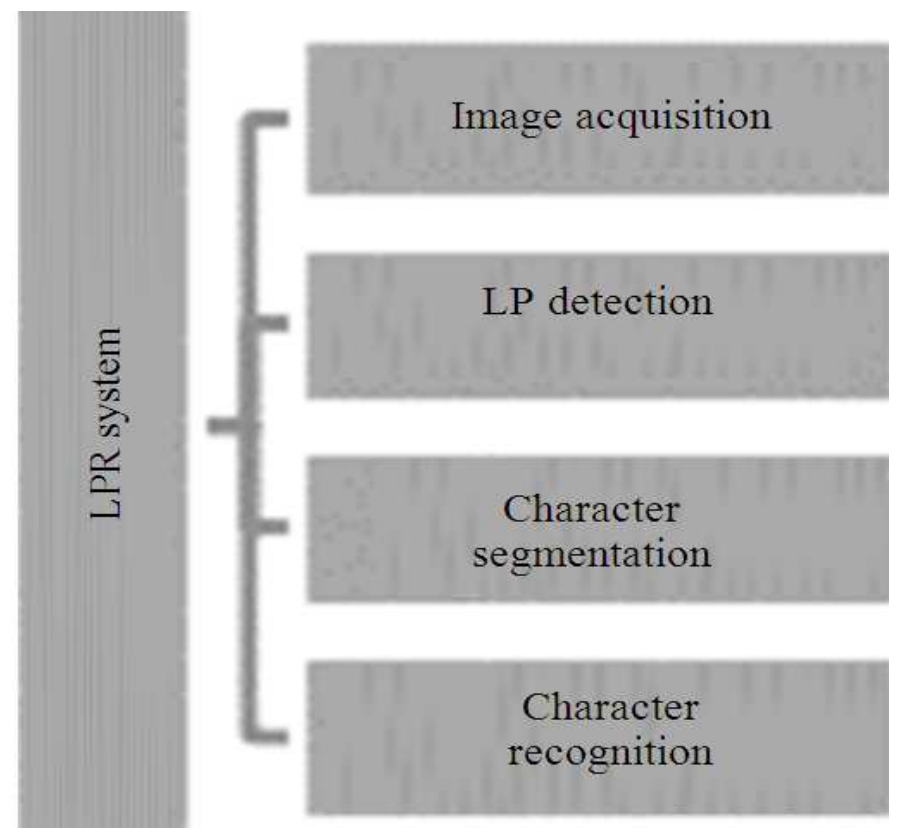

Fig. 1. Flowchart of the LPR system

In the next, this study is divided into four main sections: Related works, Proposed method, experimenatl results and conclusion.

\section{RELATED WORKS}

Generally, LP localization contains two major steps such as features extraction and termed license plate classification. In the following, we review some previous works proposed for these steps mentioned below.

\subsection{Features Extraction}

Abolghasemi and Ahmadyfard (2009) propose a mixture Gaussians functions. Two different images enhancement methods (using intensity variance and edge density) were applied in order to increase contrast of plate-like regions to avoid missing plate location especially in poor quality images. Then, authors define a novel filter which is defined as a mixture of Gaussian functions. It models the density of vertical edge at the neighborhood of plate area.

Wang et al. (2009) use a Discret Wavelet Transform based method for the license plate localization. The LP can be extracted from different qualities of source images under complex environments by using two frequency subbands. Firstly, the HL (high-low) subband is used to search the features of LP and then verify the features by checking whether in the LH (low-high) sub-band there exists a horizontal line around the feature or not.

Hough transform is another technique, which attempts to find the rectangular shapes. In our previous work (Ktata et al., 2012) we tried to select from the set of extremal regions those with shape belonging to License Plate. The detection of extremal regions can be then arranged as three steps: (a) generate a new extremal region, (b) describe the region and (c) classify it. Therefore, we define extremal regions which are connected components. The selector of the regions is implemented as a simple neural network trained on examples of regions.

Patel (2013) use a mathematical morphology in order to detect license plate. From the grayscale images, the distance between each pixel and its neighboring pixels is calculated in order to construct images edge and it can make the license plate stand out. The Sobel operator is used to extract the edge of the objects in image then apply dilation and erosion of mathematical morphology for binary images to give the image a smooth contour.

\subsection{License Plate Classification}

Neural network is widely used for plate detection and also recognition. Many classifications of the image are 
proposed and the kind of pixel is selected according to majority. In order to improve results, (Carrera et al., 2009) use a sequence of steps based on morphological operations and heuristics rules related to shape and size of the license plates.

Silva et al. (2011) presents a method using the Scale Invariant Feature Transform algorithm (SIFT) for the location of the license plate. Indeed, the two parameters position and rotation are used to filter the key points that have a realistic chance of belonging to the region of the plate. This is done by identifying the image, the areas that have features found in models corresponding to the numbers $0 \ldots 9$ in the existing license plate. After dentification number, the search begins in their neighborhood, until finding the other characters and delineating them. Finally, a character recognition algorithm based on the pixels around the transition is used for license plate recognition.

Samadi and Farhad (2011) proposes an approch based on muti-agent system for vehicule detection. Indeed, eight process agents, consisting of edge, contour, wheel, License-Plate Line (LPL), License-Plate Rectangle (LPR), Plate-Candidates Verification (PCV) and vehicle symmetry agents, were developed for vehicle detection in various outdoor scenes. Authors present some advantages of multi-agent image such as: Possibility of separated knowledge representation from different image domains, ease of construction and maintenance, heterogeneous problem solving, ability to benefit from parallel architectures and reliability.

Yi-Min et al. (2010) present a method based on AdaBoost algorithm which combined with hierarchical image and sub-window scaling schemes. The relationship is investigated among object characteristics, image structures and image scales. A parameter set is developed to easily adjust overall performance.

\section{PROPOSED METHODS}

The proposed LPD process is shown in Fig. 2. From two local databases respectively LP and non-LP a learning database is generated using Gabor filters which is introduced to a neural network for learning.

\subsection{Features Vector for Training}

In order to achieve an efficient detector with the ability to locate the plate from a vehicle image, it must cope with different variations of the plate in the vehicle image such as its position changes in direction and scale. In addition, it must be effective against changes in lighting or background.

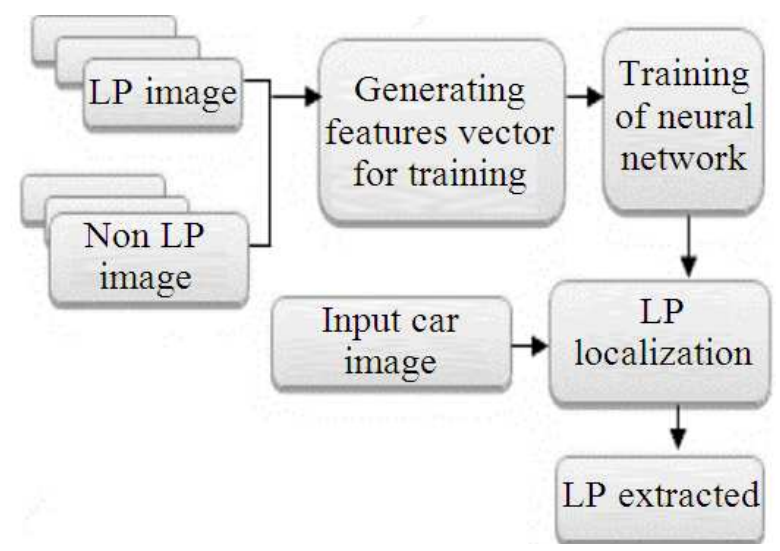

Fig. 2. License plate localization method

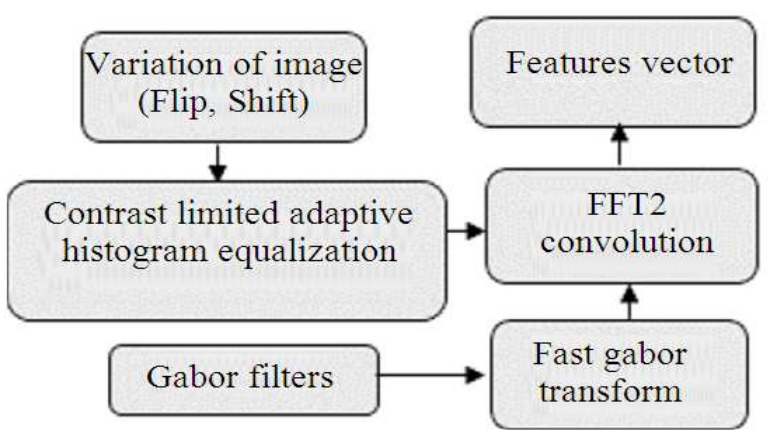

Fig. 3. Features vector for training module

The steps of generating features vector for training module, is shown in Fig. 3 above. However, these steps contain several stages.

\subsubsection{Variation of Image}

Sometimes we have to make a Customized flip horizontal or circular movement of the image matrix. This is important in order to generate the details of the features vector of the image. These variations can be used after a grey level transformation of the image.

\subsubsection{CLAHE}

The Contrast Limited Adaptive Histogram Equalization (CLAHE) is used to enhance the contrast of the image. Indeed, Contrary to Histogram, equalization works on the entire image, CLAHE operates on small regions in the image. Each region's contrast is enhanced, so that the histogram of the output region approximately matches a specified histogram. After performing the equalization, CLAHE combines neighboring tiles using bilinear interpolation to eliminate artificially induced boundaries (Garg et al., 2011). 

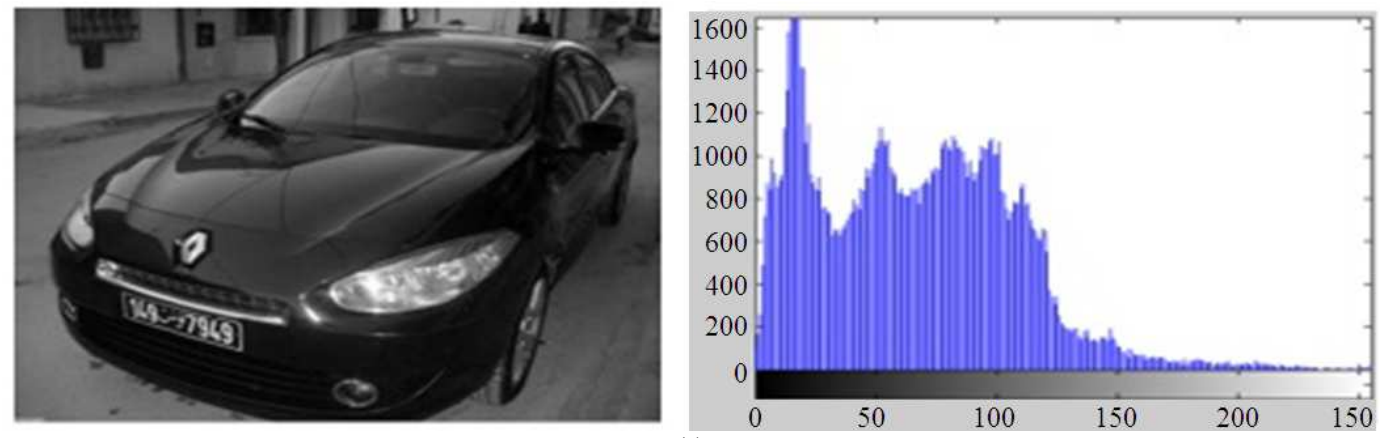

(a)
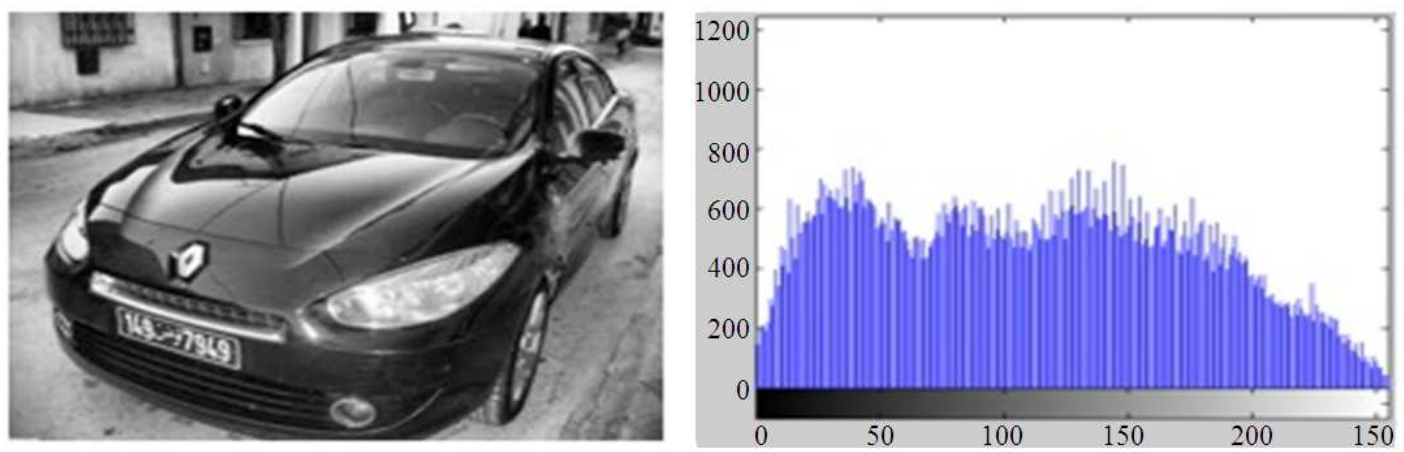

(b)
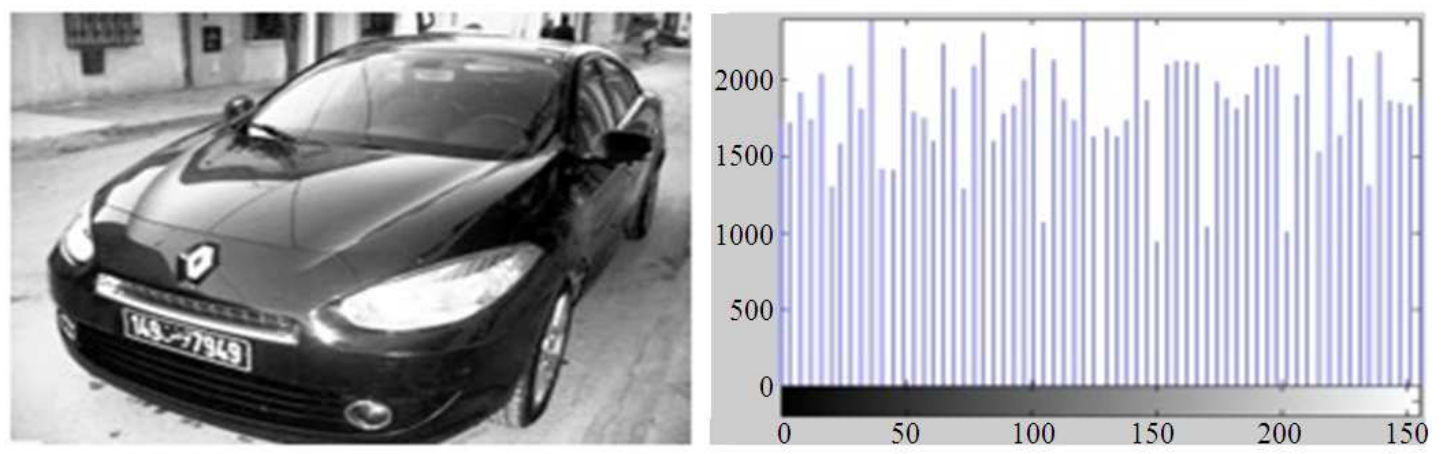

(c)

Fig. 4. Comparison of CLAHE and Histogram Equalization (a) Original image (b) CLAHE image (c) Histogram equalization image

Figure 4 shows the differences among histograms of same image, applying both methods before mentioned CLAHE and Histogram Equalization and that confirm the choice of CLAHE.

From an image, the Contrast Limited Adaptive Histogram Equalization procedure consists on:

- Divide the image into several non-overlapping regions of almost equal sizes

- Calculate the histogram of each region

- Based on a desired limit for contrast expansion, a clip limit for clipping histograms is obtained
Next, each histogram is redistributed in such a way that its height does not go beyond the clip limit.

The Equation 1 shows calculate the clip limit:

$$
\beta=(\mathrm{MN} / \mathrm{L})\left(1+\alpha / 100\left(\mathrm{~S}_{\max }-1\right)\right)
$$

Where:

$\mathrm{M}, \mathrm{N}=$ Respectively the width and height of the image

$\mathrm{L} \quad=$ The gray-scale levels

$\mathrm{S}_{\max }=$ Represents the maximum slope

a $\quad=$ The clip factor, it is a positive value between 0 and 100 


\subsubsection{Gabor Filters and Gabor Transform}

Gabor is one of the strongest tools for texture analysis which is used for license plate detection.

The arrangement of characters on license plate produces a specific texture pattern which can be considered as a plate feature. Using Gabor filters, promising results has been reported.

Gabor filters are a special class of linear filters, which are oriented filters. They allow you to highlight textures and homogeneous areas of an image. With the Gaussian filters, envelopes of filtered images provide a local spectral information at each pixel. In addition, they provide information on the energy content of the image in the direction of the selected filter Gunnar et al. (2010).

These filters have an impulse response of the form as follows Equation 2:

$$
h(x, y)=g\left(x^{\prime}, y^{\prime}\right) e^{2 \pi j\left(U_{x}+V_{y}\right)}
$$

Where:

$$
\left(x^{\prime}, y^{\prime}\right)=(x \cos \phi+y \sin \phi)+y \cos \phi
$$

The coordinates $(\mathrm{x}, \mathrm{y})$ oriented at an angle $\phi$ Equation 3:

$$
\mathrm{g}\left(\mathrm{x} ;, \mathrm{y}^{\prime}\right)=\left(1 / 2 \pi \sigma^{2}\right) \mathrm{e}^{\left(-\left(\mathrm{x}^{\prime} / \lambda\right)^{2}+\mathrm{y}^{\prime 2}\right) / 2 \sigma}
$$

The impulse response $\mathrm{h}(\mathrm{x}, \mathrm{y})$ is a complex sinusoidal function modulated by a two-dimensional Gaussian which has a ratio axis $\lambda$, expansion factor $\sigma$ and where $\phi$ is the orientation of the axis $x^{\prime}$ relative to the $\mathrm{x}$ axis. Equation 4 shows the corresponding Fourier transform:

$$
H(u, v)=e^{-2 \pi^{2} \sigma\left\{\left(u^{\prime}-U\right)^{2}+\left(v^{\prime}-v\right)^{2}\right]}
$$

Where:

$$
\left(u^{\prime}, v^{\prime}\right)=u \cos \phi+v \sin \phi-u \sin \phi+v \cos \phi
$$

$\left(\mathrm{U}^{\prime}, \mathrm{V}^{\prime}\right)$ = Obtained by rotation of the point $(\mathrm{U}, \mathrm{V})$ by the same angle $\phi$.

$\mathrm{H}(\mathrm{u}, \mathrm{v})=\mathrm{A}$ Gaussian band pass axes

$1 / \lambda$ report of a tour $\phi$ centered at $F=\sqrt{U^{2}+V^{2}}$ and angle orientation $\phi=\tan ^{-1}(\mathrm{~V} / \mathrm{U})$. We usually take $\theta=\phi$.

Well presented, filters Gabor have four degrees of freedom for extracting a portion of the spectral information of the image. This is the ratio of axes of eccentricity of the Gaussian factor in both planes and the translation imposed by $(\mathrm{U}, \mathrm{V})$ or $(\mathrm{F})$ in the frequency domain.
In this study, we use the following Equation 5 of Gabor function:

$\mathrm{g}_{\lambda, \theta, \sigma}(\mathrm{x}, \mathrm{y})=\frac{1}{2 \pi \sigma_{\mathrm{x}} \sigma_{\mathrm{y}}} \mathrm{e} \frac{-1}{2}\left[\frac{\mathrm{x}^{\prime 2}}{\sigma_{\mathrm{x}}^{2}}+\frac{\mathrm{Y}^{\prime 2}}{\sigma_{\mathrm{y}}^{2}}\right] \times \mathrm{e}_{\mathrm{e}\left[\mathrm{i} \frac{2 \pi \mathrm{x}^{\prime}}{\lambda}\right]}$

Where:

$\mathrm{X}^{\prime}=\mathrm{x} \cos \theta+\mathrm{y} \sin \theta$

$\mathrm{Y}^{\prime}=-\mathrm{x} \sin \theta+\mathrm{y} \cos \theta$

$\sigma=$ Standard deviation of the Gaussian factor

$\Lambda=$ Wavelength of the sinusoidal factor

$1 / \lambda=$ Spatial frequency of the harmonic factor

$\theta=$ Orientation of the normal to the parallel positive and negative lobes of the Gabor filters

The ratio $\sigma / \lambda$ determines the spatial frequency bandwidth of the Gabor filters. The filter responses that result from the convolution with Gabor filters are directly used as license plate detector. Five different scales and 8 directions are used, resulting in 40 Gabor filters.

The Gabor filter generated applied with the FFT I 2D in order to purge images of unwanted periodic noise. Therefore, The Fourier transform of the LP image or non-LP image will reflect the frequencies of the periodic parts of the image. By masking or filtering out theunlike frequencies one can obtain a new image by applying the inverse Fourier transformation. The filter generated is a matrix with the same dimension as the Fourier transform of the stuffed image. The components of the filter usually vary from 0 to 1 .

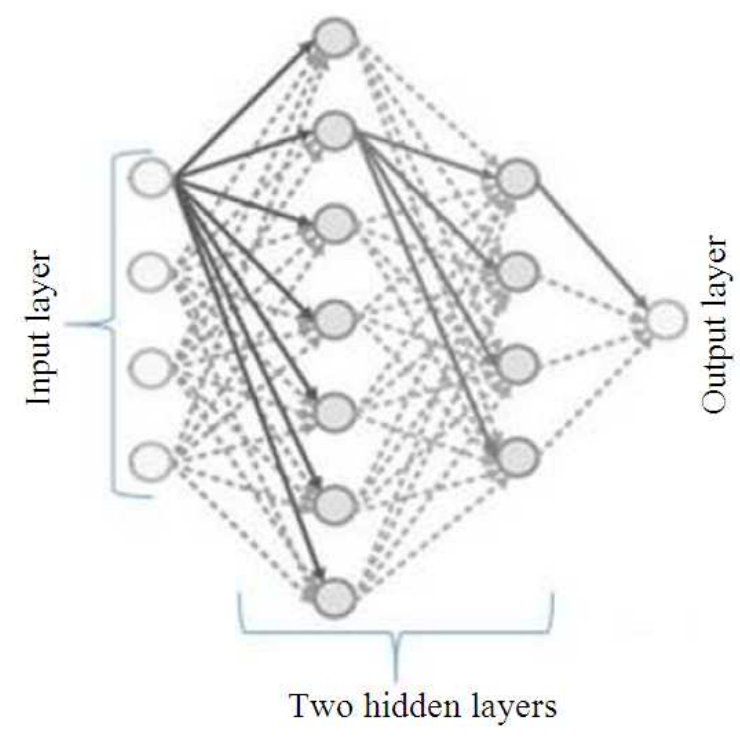

Fig. 5. Structure of MLP neural network 


\subsection{Training of Neural Network}

The system of license plate detection needs a classifier that looks at features vectors and determines if it is corresponding on LP or not. The goal is to choose a classifier able to give good results. In our method, we use a Neural Network as a classifier. Its structure is shown in which is shown in Fig. 5 above.

The classifier has 17889 inputs that correspond to the zone descriptors generated using gabor filters and it has one output 0 or 1 depending whether the window analyzed belongs or not to a zone of the license plate. A neural classifier has been chosen because this network is a universal function estimator. The network is trained with the scaled conjugate gradient back-propagation function. This method was chosen because it gives criteria to determine the numbers of neurons of the hidden layer and includes a regularization strategy that permits an appropriate generalization.
The MLP Network implemented for our method is composed of three layers, one input, one hidden and one output.

\subsection{License Plate Localization}

In order to identify the LP, for testing, each image is scanned at all possible locations and scales by a subwindow (patch). Each patch is fed to the Gabor function and the output vector goes to the classifier. There areways to pre-select possible locations and how to pin point the location of the license plate.

\section{EXPERIMENTAL RESULTS}

In the experiments, the proposed method is evaluated on an a PC with $2 \times 2.4 \mathrm{GHz}$ CPU, $3 \mathrm{~GB}$ RAM and Windows 7 operating system. For the training stage we have used 120 images (bmp and jpeg format) of $100 \times 40$ pixels of LP and 38 images of $100 \times 40$ pixels of non LP.
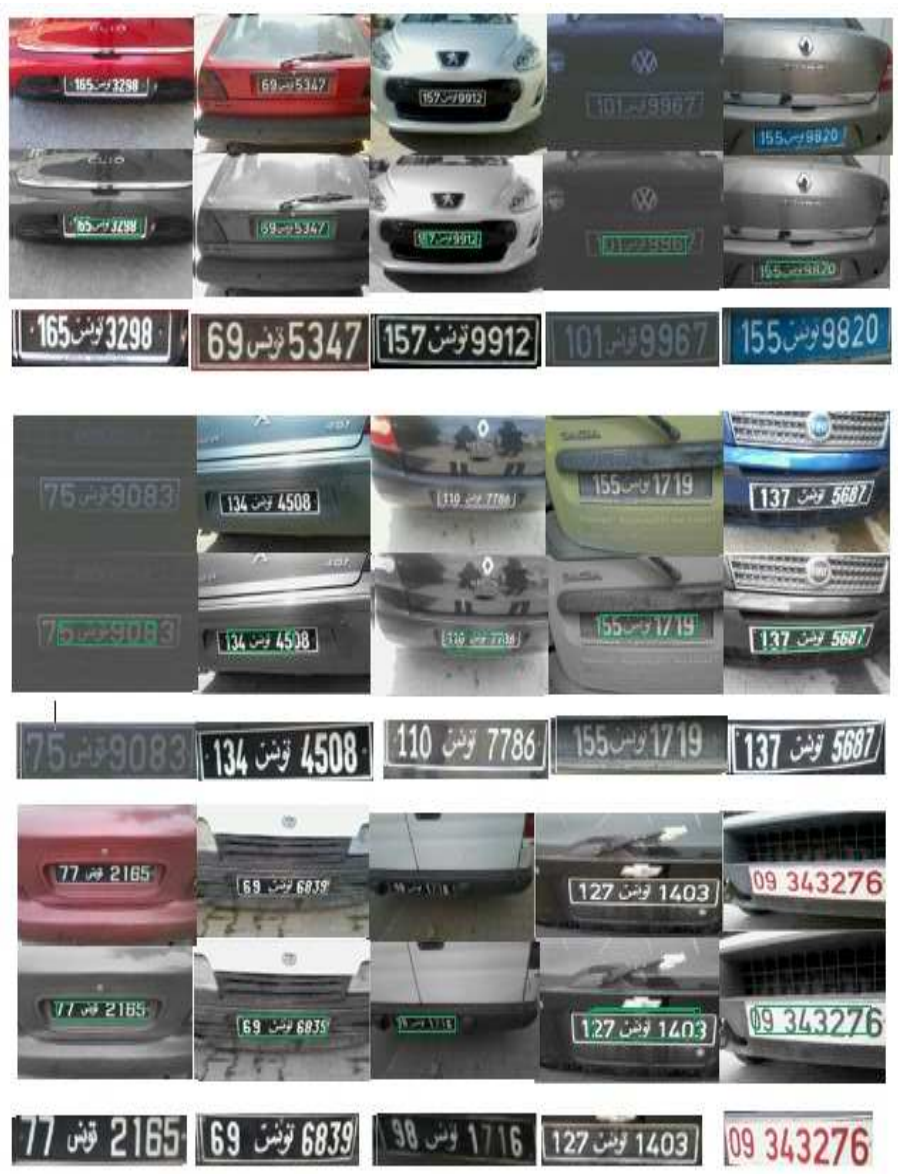

Fig. 6. Localization and extraction of Tunisian license plate's samples 
The test image set includes 58 different images with each of size $320 \times 240$.

The Fig. 6 shows images samples and their detected license plates. The experimental results show that the proposed algorithm can detect the location of LP in some environments with acceptable accuracy.

\section{CONCLUSION}

In this study, we have presented an approach for License plate detection. The LP detection system presents three steps: Generating vector of training data, Training of Neural network and LP Localization. Our approachincludes a descriptor extraction based on Gabor Filters. Our approach was applied to a simple image of license plate. A known disadvantage of the current method is that the configurable parameters don't contain external conditions that influence LP images, for example, LP images taken in raining or snowy day, partial LP occlusion and others. Therefore, future works include improvements of the configurable parameters for external conditions and algorithm to development of LP recognition module other classifiers.

\section{REFERENCES}

Abolghasemi, V. and A. Ahmadyfard, 2009. An edgebased color-aided method for license plate detection. Image Vis. Comput., 27: 1134-1142. DOI: 10.1016/j.imavis.2008.10.012

Carrera, L., M. Mora, J. Gonzalez and F. Aravena, 2009. License plate detection using neural networks. Lecture Notes Comput. Sci., 5518: 1248-1255. DOI: 10.1007/978-3-642-02481-8_186
Garg, R., B. Mittal and S. Garg, 2011. Histogram equalization techniques for image enhancement. Int. J. Electron. Commun. Technol., 2: 107-111.

Gunnar, L. C. Olivier, K. Hans and B. Magnus, 2010. Non-ring filters for robust detection of linear structures. Proceedings of the 20th International Conference on Pattern Recognition, (PR' 10), IEEE Computer Society Washington, DC., pp: 233-236. DOI: $10.1109 /$ ICPR.2010.66

Ktata, S., F. Benzarti and H. Amiri, 2012. License plate detection using hough transforms. Proceedings of the 2nd Workshop of Signal and Document Processing, Mar. 23-24, Hammamet Tunisia.

Patel, S.G., 2013. Vehicle license plate recognition using morphology and neural network. Int. J. Cybernetics Inform.

Samadi, S. and M. Farhad, 2011. A multi-agent visionbased system for vehicle detection. World Applied Sci. J., 15: 1722-1732.

Silva, F.A., A.O. Artero, M.S.V. Paiva and R.L. Barbosa, 2011. ALPRs-A new approach for license plate recognition using the SIFT algorithm. Signal Image Proc. Int. J., 4: 17-17.

Wang, Y.R. W.H. Lin and S.J. Horn, 2009. Fast license plate localization using discrete wavelet transform. Lecture Notes Comput. Sci., 5574: 408-415. DOI: 10.1007/978-3-642-03095-6_39

Yi-Min, T., H. Keng-Yen, T. Chih-Chung and C. LiangGee, 2010. An exploration of on-road vehicle detection using hierarchical scaling schemes. Proceedings of 2010 IEEE 17th International Conference on Image Processing, Sept. 26-29, IEEE Xplore Press, Hong Kong, pp: 3937-3940. DOI: 10.1109/ICIP.2010.5649931 